\title{
THE VOLUME OF A REGION DEFINED BY POLYNOMIAL INEQUALITIES
}

\author{
O. S. ROTHAUS ${ }^{1}$
}

\begin{abstract}
Let $P(x)$ be a polynomial on $\boldsymbol{R}^{n}$ with nonnegative coefficients. We develop a simple necessary and sufficient condition that the set $S=\left\{x \in \mathbb{R}^{n} \mid x_{i} \geqq 0, P(x) \leqq 1\right\}$ shall have finite volume. A corresponding result where $P(x)$ is replaced by a collection of polynomials is an easy corollary. Finally, the necessary and sufficient conditions for the special case that $P$ is a product of linear forms is also given.
\end{abstract}

Let $P(x)$ be a polynomial on $\boldsymbol{R}^{n}$ with nonnegative coefficients, and without constant term (to avoid trivial complications).

$$
P(x)=\sum_{v=1}^{k} r_{v} x_{1}^{c_{v(1)}} x_{2}^{c_{v}(2)} \cdots x_{n}^{c_{\tau}(n)}, \quad r_{v}>0 .
$$

The vectors $c_{v}=\left(c_{v(1)}, c_{v(2)}, \cdots, c_{v(n)}\right)$ are called the exponents of $P$. Let $C$ be the closed convex cone in $\boldsymbol{R}^{n}$ generated by the $c_{v}$, i.e., the elements of $C$ are all linear combinations $p_{1} c_{1}+p_{2} c_{2}+\cdots+p_{k} c_{k}$ with $p_{i} \geqq 0$. Let $\langle$,$\rangle be the usual inner product in R^{n}$, and let $C^{*}$ be the dual cone to $C$ with respect to this scalar product; i.e., $C^{*}$ is the set of $y \in R^{n}$ such that $\langle y, x\rangle \geqq 0 \forall x \in C$. Note that $C^{*}$ contains the first $2^{n}$-gant in $\boldsymbol{R}^{n}$, so $C^{*}$ has nonempty interior.

There are several well-known features of the above situation, which it is easy to establish using separation properties of convex sets. Thus if $b$ is not an interior point of $C$, there exists $d \in C^{*}, d \neq 0$, such that $\langle d, b\rangle \leqq 0$. While if $b \neq 0$ is an interior point of $C$, then there exists a positive constant $p$ such that $\langle d, b\rangle \geqq p\langle d, d\rangle^{1 / 2} \forall d \in C^{*}$, as an easy compactness argument shows. Then we have

THEOREM 1. The set $S=\left\{x \mid x_{i} \geqq 0, P(x) \leqq 1\right\}$ is of finite volume if and only if the vector $m=(1,1, \cdots, 1)$ is an interior point of $C$. (In particular, $C$ must have a nonempty interior.)

Received by the editors February 26, 1973.

AMS (MOS) subject classifications (1970). Primary 52A20; Secondary 10E05, $10 \mathrm{E} 15$.

${ }^{1}$ Work partially supported by NSF GP8129. 
Proof. ( $S$ is convex, but we do not need this fact.)

$$
\text { Vol } S=\int_{x_{i} \geqq 0 . P(x) \leqq 1} d x=\int_{P\left(e^{-u}\right) \leqq 1} e^{-\langle m \cdot u\rangle} d u .
$$

Now pick a vector $y$ such that $\left\langle c_{v}, y\right\rangle \geqq \log k r_{v}$. Then if $u \in C^{*}+y$,

$$
P\left(e^{-u}\right)=\sum_{v=1}^{k} r_{v} e^{-\langle c v . u\rangle} \leqq \sum_{v} r_{v} \frac{1}{k r_{v}}=1 .
$$

So $\left\{u \in R^{n} \mid P\left(e^{-u}\right) \leqq 1\right\} \subset C^{*}+y$.

Also pick a vector $w$ such that $\left\langle c_{v}, w\right\rangle \leqq \log r_{v}$. Then if $P\left(e^{-u}\right) \leqq 1$, we must have $r_{v} e^{-\left\langle c_{v} \cdot u\right\rangle} \leqq 1$, which implies that $\left\langle c_{v}, u\right\rangle \geqq \log r_{v}$, which implies that $\left\langle c_{v}, u-w\right\rangle \geqq 0$, i.e., $u \in w+C^{*}$.

Thus the set $\left\{u \in R^{n} \mid P\left(e^{-u}\right) \leqq 1\right\}$ is contained in some translate of $C^{*}$, and contains a second translate. It follows that Vol $S$ is finite if and only if $\int_{C} e^{-\langle m, u\rangle} d u$ is finite. But if $m$ is an interior point of $C$, then $\langle m, u\rangle \geqq$ $p\langle u, u\rangle^{1 / 2}$ for $u \in C^{*}$, and the integral is obviously finite. While if $m$ is not an interior point, it is easy to see that the above integral diverges, completing the proof.

COROllary. Let $P_{1}, P_{2}, \cdots, P_{r}$ be polynomials on $R^{n}$ with nonnegative coefficients. The set

$$
S=\left\{x \mid x_{i} \geqq 0, P_{1}(x) \leqq 1, P_{2}(x) \leqq 1, \cdots, P_{r}(x) \leqq 1\right\}
$$

is of finite volume if and only if $m=(1,1, \cdots, 1)$ is an interior point of the cone generated by the exponents of all the polynomials $P_{i}$.

For if $x \in S$, then $r^{-1} P_{1}(x)+r^{-1} P_{2}(x)+\cdots+r^{-1} P_{r}(x) \leqq 1$, while if $P_{1}(x)+P_{2}(x)+\cdots+P_{r}(x) \leqq 1, x \in S$.

Next, we apply the above theorem to the case when $P(x)$ is a product of linear forms on $\boldsymbol{R}^{n}$.

$$
P(x)=\prod_{v=1}^{k}\left(a_{v(1)} x_{1}+a_{v(2)} x_{2}+\cdots+a_{v(n)} x_{n}\right),
$$

each linear form having nonnegative coefficients not all zero. Let $U$ be a subset of $\{1,2, \cdots, n\}$. We say that the support of the linear form $a_{1} x_{1}+a_{2} x_{2}+\cdots+a_{n} x_{n}$ is $U$ if $a_{i} \neq 0$ for $i \in U$, and $a_{i}=0$ for $i \notin U$. For any subset $U$, let $N(U)$ be the number of linear forms in product for $P(x)$ whose support is contained in $U$. Then we have:

THEOREM 2. Vol $S$ is finite if and only if for every proper subset $U$, we have $N(U) /$ card $U<k / n$. 
To prove the "if" part, let $u=\left(u_{1}, u_{2}, \cdots, u_{n}\right) \in C^{*}$, and suppose without loss of generality that $u_{1} \geqq u_{2} \geqq \cdots \geqq u_{n}$. For $1 \leqq r \leqq n$, put $N_{r}=N(\{1,2, \cdots, r\})$. Then the vector $c=\left(N_{1}, N_{2}-N_{1}, N_{3}-N_{2}, \cdots\right.$, $\left.N_{n}-N_{n-1}\right)$ is an exponent of $P$.

Hence

$$
\begin{aligned}
\langle c, u\rangle & =N_{1}\left(u_{1}-u_{2}\right)+N_{2}\left(u_{2}-u_{3}\right)+\cdots+N_{n-1}\left(u_{n-1}-u_{n}\right)+k u_{n} \\
& \leqq(k / n)\left(u_{1}+u_{2}+\cdots+u_{n}\right)
\end{aligned}
$$

with equality if and only if $u_{1}=u_{2}=\cdots=u_{n}$. Since $\langle c, u\rangle \geqq 0$, we obtain $\langle m, u\rangle>0$ if the components of $u$ are not all equal. While if the components of $u$ are all equal and not all zero, then since $u \in C^{*}$, the components of, $u$ are all positive, and again $\langle m, u\rangle>0$. This proves that $m$ is an interior point of $C$, and completes the proof of "if".

For the "only if" part, suppose that, for $U=\{1,2, \cdots, r\}, N(U) / r \geqq k / n$. We will show $m$ cannot be $a n$ interior part of $C$. Consider the vector $u$ whose first $r$ components are equal to $n-r$, and whose remaining $n-r$ components are equal to $-r$. For any exponent $c=\left(c_{1}, c_{2}, \cdots, c_{n}\right)$, we have

$$
\begin{aligned}
\langle c, u\rangle & =\left(c_{1}+c_{2}+\cdots+c_{r}\right)(n-r)-\left(c_{r+1}+\cdots+c_{r}\right) r \\
& =\left(c_{1}+c_{2}+\cdots+c_{r}\right) \cdot n-k r .
\end{aligned}
$$

As $c$ runs through all exponents of $P,\langle c, u\rangle$ will be minimum when $c_{1}+c_{2}+\cdots+c_{r}$ is as small as possible, i.e., when $c_{1}+c_{2}+\cdots+c_{r}=N(U)$. Since $N(U) \geqq k r / n$, we have always $\langle c, u\rangle \geqq 0$ for any exponent $c$. Hence $u \in C^{*}$; but $\langle m, u\rangle=0$ and this proves $m$ is not an interior point of $C$, and completes the proof.

Department of Mathematics, Cornell University, Ithaca, New York 14850

Department of Mathematics, The Hebrew University, Jerusalem, Israel 